\title{
O Metanol e as Células de Combustível
}

\section{Introdução}

O princípio de funcionamento das células de combustível foi experimentalmente demonstrado por Sir William Grove ${ }^{1}$ já em 1839 quando os níveis de poluição sonora e atmosférica não haviam ainda atingido os valores elevados que hoje são detectados, particularmente em meios urbanos e áreas industriais.

Efectivamente William Grove observou que da electrólise de uma solução aquosa de $\mathrm{H}_{2} \mathrm{SO}_{4}$ com eléctrodos de Platina resultava $\mathrm{O}_{2}$ e $\mathrm{H}_{2}$ electroquimicamente activos, originando uma célula com o potencial de $1 \mathrm{~V}$, em circuito aberto. Contudo, a densidade de corrente produzida era tão pequena que a referida célula não teve qualquer utilidade prática. Porém a ideia estava lançada: é possivel tranformar directamente energia quimica em energia eléctrica, utilizando como combustíveis electroquímicos o $\mathrm{H}_{2}$ e o $\mathrm{O}_{2}$ do ar, sem produzir ruído, nem poluição atmosférica.

\section{Células $\mathrm{H}_{2} / \mathrm{O}_{2}$}

$\mathrm{Na}$ figura 1 representa-se esquematicamente uma célula de $\mathrm{H}_{2} / \mathrm{O}_{2}$ (ar).

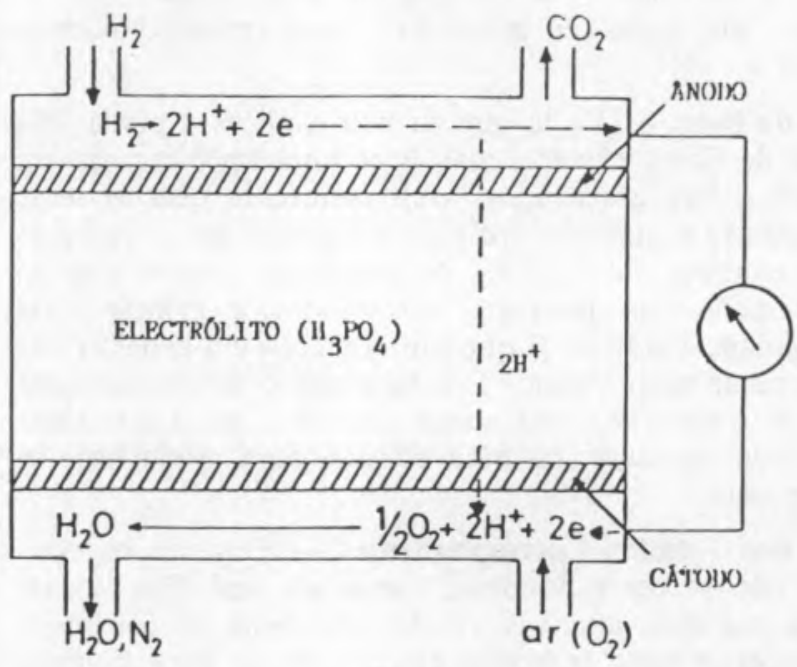

Fig. 1

Representação esquemática de uma célula de combustivel.

$\mathrm{O} \mathrm{H}_{2}$ funciona como combustível anódico e o $\mathrm{O}_{2}$ como combustível catódico, de acordo com as reacçðes descritas por,

$\mathrm{H}_{2} \rightarrow 2 \mathrm{H}^{+}+2 \mathrm{e}$

e

$1 / 2 \mathrm{O}_{2}+2 \mathrm{H}^{+}+2 \mathrm{e} \rightarrow \mathrm{H}_{2} \mathrm{O}$ ou ainda,

$\mathrm{H}_{2}+1 / 2 \mathrm{O}_{2} \rightarrow \mathrm{H}_{2} \mathrm{O}$

Sendo a Energia de Gibbs associada ao processo (3) negativa resulta naturalmente produção espontânea de energia eléctrica (fluxo de electrōes), quando os dois eléctrodos (ânodo e cátodo) forem ligados a uma circuito exterior.

A força electromotriz da célula descrita por (1) e (2), admitindo um comportamento reversível do sistema, deverá ser de aproximadamente $1 \mathrm{~V}$. Contudo, tal só se verifica na prática para temperaturas $>150^{\circ} \mathrm{C}$.

Como produto da reacção química que origina a corrente eléctrica tem-se apenas $\mathrm{H}_{2} \mathrm{O}$ (reacção 3 ), o que significa que as referidas células são meios potenciais de produção de energia eléctrica, sem produção de ruído, nem emissão de gases poluentes, constituindo uma forma de energia "limpa». As combustões são aqui substituídas por processos de eléctrodo. A célula só consome combustível quando fornece corrente eléctrica ao exterior.

Porque não há perdas inerentes ao ciclo de Carnot, uma célula de $\mathrm{H}_{2} / \mathrm{O}_{2}$ deveria, pelo menos em teoria, atingir eficiências da ordem de $96 \%$. Contudo, tais níveis de eficiência são, ainda hoje, inatingiveis, como se pode concluir da Tabela 1. Porém, os valores já atingidos (38\%-55\%) são promissores, quando comparados com as eficiências globais das estaçð̃es piloto de carvão ( $40 \%$ de eficiência), geradores eléctricos a diesel $(28 \%)$ ou ainda, motores a gasolina $(12 \%)$.

$\mathrm{O}_{2}$ é um combustível óptimo por reagir de um modo eficiente, a temperaturas próximas da ambiente, em particular nos substratos Platina/«Negro de Platina»; porém é um gás e como tal, difícil de transportar. Assim, ou é armazenado criostaticamente no estado líquido (tal como aconteceu nas missð̃es Apolo), ou é armazenado em cilindros de transporte difícil e perigoso, já que $\mathrm{o} \mathrm{H}_{2}$ é um gás facilmente inflamável.

TABELA 1

Parâmetros característicos de células de combustivel

\begin{tabular}{llccc}
\hline Combustiveis & Electrólito & $\begin{array}{c}\text { Densidade } \\
\text { de corrente } \\
\left(\mathrm{mA} \mathrm{cm} \text { cm }^{-2}\right)\end{array}$ & $\begin{array}{c}\text { Potencial } \\
\text { de célula } \\
\text { (V) }\end{array}$ & $\begin{array}{c}\text { Eficiência } \\
(\%)\end{array}$ \\
\hline $\mathrm{H}_{2}-$ ar & $\mathrm{H}_{2} \mathrm{SO}_{4}$ (aq.) & 200 & 0,65 & 43 \\
$\mathrm{H}_{2}-\mathrm{O}_{2}$ & $\mathrm{KOH}_{\text {(aq.) }}$ & 400 & 0,8 & 55 \\
$\mathrm{CH}_{3} \mathrm{OH}$-ar & $\mathrm{H}_{3} \mathrm{PO}_{4}$ (aq.) & 50 & 0,5 & 38 \\
\hline
\end{tabular}

a Dep. de Química da Faculdade de Ciências da Universidade de Lisboa CECUL - Rua da Escola Politécnica, 58, 1294 LISBOA CODEX. 


\section{Os Hidrocarbonetos como alternativa ao $\mathbf{H}_{2}$}

Os hidrocarbonetos naturais surgem então como combustível anódico para substituir $\mathrm{o}_{2} \mathrm{H}_{2}$ nas células de $\mathrm{H}_{2} / \mathrm{O}_{2}$. Os hidrocarbonetos por reacção com água, na presença de bons catalisadores e a temperaturas elevadas $\left(\mathrm{t}>750^{\circ} \mathrm{C}\right)$ produzem uma mistura gasosa rica em $\mathrm{H}_{2}$. A referida mistura contém porém, $\mathrm{CO}_{2}$ e algum $\mathrm{CO}$, este resultante do equilíbrio,

$$
\underset{\text { (gás) }}{\mathrm{CO}_{2}}+\underset{\text { (gás) }}{\mathrm{H}_{2}} \rightleftarrows \underset{\text { (gás) }}{\mathrm{CO}}+\underset{\text { (vapor) }}{\mathrm{H}_{2} \mathrm{O}}
$$

A concentração em $\mathrm{CO}$ na mistura, é tanto maior quanto mais elevada for a temperatura a que decorre a transformação do hidrocarboneto. A presença de $\mathrm{CO}_{2}$ e CO na mistura implica a eliminação de $\mathrm{CO}$ e também a substituição dos electrólitos alcalinos por electrólitos ácidos de modo a obviar a formação de carbonatos (produtos não desejáveis!).

Ao substituir-se o electrólito alcalino por um ácido, os materiais de eléctrodo poderão ter que ser substituídos por outros mais resistentes à corrosão, e em princípio mais caros. A operação de eliminação do $\mathrm{CO}$ tem que ser introduzida no processo, já que este conduz à perda de actividade do substrato. A inclusão daquela operação torna necessariamente o sistema mais complexo e dispendioso. O processo como um todo envolve pelo menos 3 reactores a funcionar a temperaturas bastante diferentes, respectivamente, $750^{\circ} \mathrm{C} ; 300-450^{\circ} \mathrm{C}$ e $250-155^{\circ} \mathrm{C}$.

\section{O Metanol como combustível alternativo aos Hidro- carbonetos}

O metanol, derivado em grande parte do gás natural pode ser facilmente convertido em $\mathrm{H}_{2}$ e $\mathrm{CO}_{2}$, de acordo com a reacção descrita por,

$\mathrm{CH}_{3} \mathrm{OH}+\mathrm{H}_{2} \mathrm{O} \rightarrow \mathrm{CO}_{2}+3 \mathrm{H}_{2}$

A reacção decorre a temperaturas não muito elevadas $\left(250-400^{\circ} \mathrm{C}\right)$ desde que seja utilizado um catalisador apropriado.

De acordo com (5) não há produção de $\mathrm{CO}$, pelo que a etapa de purificação da mistura é naturalmente eliminada. O metanol apresenta-se como um combustivel líquido e solúvel em meio aquoso à temperatura ambiente. Em solução aquosa o Metanol origina por via electroquímica iðes $\mathrm{H}^{+}$, de acordo com a reacção descrita por,

$$
\mathrm{CH}_{3} \mathrm{OH}+\mathrm{H}_{2} \mathrm{O} \rightarrow 6 \mathrm{H}^{+}+\mathrm{CO}_{2}+6 \mathrm{e}
$$

Diferentes protótipos de células utilizando como combustível anódico o Metanol, têm sido fabricadas numa tentativa de optimizar as suas características e de as tornar competitivas. Contudo, o preço de produção não é ainda competitivo, o que não invalida a sua utilidade e aplicação em vários domínios, mais que não fosse pelo facto de serem agentes não poluentes.

A tabela 2 sumariza algumas das características de dois protótipos de células que utilizam o metanol como combustível anódico. Um número apreciável de sistemas piloto com capacidade de vários KW tem sido testado, em especial no Japão e nos E.U.A, tendo provado funcionar bem e com tempos de vida a excederem as $10000 \mathrm{~h}$.
TABELA 2

\begin{tabular}{|c|c|c|c|c|c|}
\hline Fabricante & Electrólito & $\begin{array}{c}\text { Tempera- } \\
\text { tura } \\
\left({ }^{\circ} \mathrm{C}\right)\end{array}$ & Ânodo & $\begin{array}{c}\text { Densidade } \\
\text { de } \\
\text { corrente } \\
\left(\mathrm{mA} \mathrm{cm} \mathrm{cm}^{-2}\right)\end{array}$ & $\begin{array}{l}\text { Potencial } \\
\text { (V) }\end{array}$ \\
\hline $\begin{array}{l}\text { Shell Res. } \\
\text { Ltd.U.K. }\end{array}$ & $\mathrm{KOH}$ & 65 & $\begin{array}{l}\text { Plástico } \\
\text { poroso } \\
\text { PVC }(5 \mu) \text {, } \\
\text { superficie } \\
\text { metalizada } \\
\text { com Ag e } \\
\text { activada } \\
\text { com Pt } \\
1 \mathrm{mg} \mathrm{cm}{ }^{-2}\end{array}$ & 60 & $0,6-0,7$ \\
\hline $\begin{array}{l}\text { Esso Res. } \\
\text { \& Eng. } \\
\text { Co.U.S.A. }\end{array}$ & $\begin{array}{l}3.7 \mathrm{M} \\
\mathrm{H}_{2} \mathrm{SO}_{4} \\
+0,75 \mathrm{M} \\
\mathrm{CH}_{3} \mathrm{OH}\end{array}$ & $60-80$ & $\begin{array}{l}20 \mathrm{mg} \text { de } \\
\mathrm{Pt} / \mathrm{cm}^{-2} \\
\text { de Tân- } \\
\text { talo }\end{array}$ & 40 & 0,4 \\
\hline
\end{tabular}

Características de dois prototipos de células que utilizam o Metanol como combustivel anódico

Contudo, um certo número de problemas carece de solução, para que as referidas células atinjam sucesso comercial. Os parâmetros a optimizar dependem dos fins a que se destinam as células, como se pode concluir de apenas dois exemplos que a Tabela 3 apresenta.

TABELA 3

Especificações exigidas para as células de combustível de acordo com a sua possível aplicação

\begin{tabular}{lll}
\hline & $\begin{array}{c}\text { Tracção de um } \\
\text { automóvel }\end{array}$ & $\begin{array}{c}\text { Auxiliar do funcionamento } \\
\text { do coração «Pacemaker» }\end{array}$ \\
\hline $\mathrm{V}_{\text {cel }}$ & $90-400 \mathrm{~V}$ & $1-3 \mathrm{~V}$ \\
Capacidade & $10-20 \mathrm{KAh}$ & $0.5 \mathrm{KAh}$ \\
Tamanho & $0.1-0.5 \mathrm{~m}^{3}$ & alguns $\mathrm{cm}^{3}$ \\
Temperatura & $250-313^{\circ} \mathrm{K}$ & $310^{\circ} \mathrm{K}$ \\
Custo & $\begin{array}{l}\text { menor ou equivalente } \\
\text { à gasolina }\end{array}$ & pouco importa \\
\hline
\end{tabular}

Há porém, parâmetros que seja qual for a aplicação interessa optimizar, nomeadamente:

(i) potencial ou «tensão» da célula, $V_{\text {cel }}$

(ii) densidade de corrente, $\mathrm{i}_{\max }$

(iii) eficiência efectiva

e

(iv) custo.

A optimização de qualquer dos parâmetros interfere com os outros, dada a sua interdependência.

A Tabela 4 indica a meta a atingir na optimização.

TABELA 4

Valores já obtidos e a obter para uma optimização das células de combustivel

\begin{tabular}{lll}
\hline & Valores já obtidos & Valores a obter \\
\hline $\mathrm{V}_{\text {cel }}$ & 0,6 a $0,85 \mathrm{~V}$ & 1,2 a $1,4 \mathrm{~V}$ \\
$\mathrm{i}_{\max }$ & 50 a $150 \mathrm{~mA} \mathrm{~cm}^{-2}$ & $200-500 \mathrm{~mA} \mathrm{~cm}$ \\
Eficiência & 35 a $47 \%$ & 80 a $100 \%$ \\
\hline
\end{tabular}

Os esforços iniciados nos anos 60 neste domínio continuam hoje a merecer a dedicação de equipas de investigadores quer a nível de investigação fundamental dirigida, quer a nível de estaçðes piloto.

A Comissão das Comunidades Europeias iniciou recentemente um projecto que visa o desenvolvimento das 
células de combustível de metanol/ar, como parte do programa «Non Nuclear Energy Research and Development». Os objectivos a atingir são uma potência de $500 \mathrm{~W} / \mathrm{cm}^{2}$ com um catalisador em que a dispersão do metal nobre seja $1 \mathrm{mg} \mathrm{cm}^{-2}$ para uma temperatura de funcionamento de $65^{\circ} \mathrm{C}$.

\section{Oxidação Anódica do Metanol}

Em aplicaçðes industriais interessa obter densidades de corrente elevadas (pelo menos $>100 \mathrm{~mA} \mathrm{~cm}-2$ ), com sobrepotenciais pequenos, idealmente próximos do potencial reversível. Interessa pois estudar a cinética dos processos de eléctrodo, de modo a torná-los mais rápidos e eficientes, o que significa reduzir o sobrepotencial e/ou aumentar a densidade de corrente. Por outro lado, interessa também procurar substratos (eléctrodos), em que o processo mantenha as características cinéticas ideais a um custo mais baixo.

Em geral, os processos que vimos referindo só ocorrem a uma velocidade apreciável a temperaturas baixas, quando o substrato é a Platina ou um metal «nobre» como se conclui da análise da Tabela 5 .

TABELA 5

Efeito do substrato nos parâmetros cinéticos da oxidação electroquimica do metanol

\begin{tabular}{lcc}
\hline Substrato & $\mathrm{i}_{\mathrm{o}}^{*}\left(\mathrm{~A} \mathrm{~cm}^{-2}\right)$ & Inclinação de Tafel/V \\
\hline $\mathrm{Pt}-\mathrm{Fe}$ & $1 \times 10^{-4}$ & 0.103 \\
$\mathrm{Pt}-\mathrm{Ni}$ & $5 \times 10^{-5}$ & 0.109 \\
$\mathrm{Pt}-\mathrm{Co}$ & $3.2 \times 10^{-6}$ & 0.084 \\
$\mathrm{Pt}-\mathrm{Pb}$ & $1.0 \times 10^{-6}$ & 0.085 \\
$\mathrm{Pt}$ & $5 \times 10^{-8}$ & 0.066 \\
\hline
\end{tabular}

* $\mathrm{i}_{\mathrm{o}}$ representa a densidade de corrente de troca para $\eta=0$, ou seja, para $\mathrm{E}=\mathrm{E}_{\mathrm{rev}}$.

Uma das razסes que certamente contribui para que a cinética do processo descrito por (6) seja lenta e o sobrepotencial elevado, é o facto de o processo envolver vários passos, de modo a trocar os 6 electrőes. Há pois, que conhecer o mecanismo conducente aos produtos finais, identificar as espécies intermediárias de modo a concluir sobre como modificar o substrato transformando-o num bom catalisador do processo global. De um modo análogo se deve proceder em relação ao processo catódico de redução do $\mathrm{O}_{2}$, já que

$\mathrm{V}_{\text {célula }}=\mathrm{E}_{\mathrm{rev}}^{\mathrm{c}}-\mathrm{E}_{\mathrm{rev}}^{\mathrm{a}}-\left|\eta_{\mathrm{a}}\right|-\left|\eta_{\mathrm{c}}\right|-(\text { IR })_{\text {célula }}$.

Interessa pois minimizar quer o sobrepotencial anódico $\eta_{\mathrm{a}}$, quer o catódico $\eta_{\mathrm{c}}$, bem como a queda Óhmica IR. A diminuição da parcela IR é conseguida à custa de:

- selecção do electrólito (condutibilidade elevada, de modo a reduzir $\mathrm{R}$ )

- separadores quando existirem de resistência baixa, e - design convenientemente estudado.

A literatura apresenta depois dos anos 70 resultados de projectos de investigação sobre a oxidação do metanol em diferentes substratos como por ex. ligas metálicas em vez de apenas $\mathrm{Pt}^{2-3}$, ou mais recentemente o substrato $\mathrm{Pt}$ modificado pela deposição de adatomos de metais, como o $\mathrm{Ru}$, o $\mathrm{Sn}$ e $\circ \mathrm{Ge}^{4},{ }^{5}$, ou ainda outros como o $\mathrm{Bi}, \mathrm{Cd}, \mathrm{Tl}$ ou $\mathrm{Pb}^{6-8}$.

$\mathrm{O}$ efeito catalítico referente à oxidação "anódica» do metanol, conseguido com a adição de pequenas quanti- dades de ið̃es metálicos, é demonstrado pelas curvas I-E da fig. 2 e pelos valores da Tabela 6 .

TABELA 6

Efeito da adição de iões metálicos na oxidação do Metanol (ref. 7)

\begin{tabular}{lc}
\multicolumn{1}{c}{ Sistemas } & $10^{10} \mathrm{i}_{\mathrm{o}} / \mathrm{A} \mathrm{cm}^{-2}$ \\
\hline $\mathrm{Pt} / \mathrm{CH}_{3} \mathrm{OH} 0.15 \mathrm{M}+0,2 \mathrm{M} \mathrm{NaOH}$ & 2.8 \\
$\mathrm{Pt} / \mathrm{Tl}^{+}\left(1 \times 10^{-6} \mathrm{M}\right)+\mathrm{CH}_{3} \mathrm{OH} 0.15 \mathrm{M}+0,2 \mathrm{M} \mathrm{NaOH}$ & 7.5 \\
$\mathrm{Pt} / \mathrm{HPbO}_{2}^{-}\left(5 \times 10^{-6} \mathrm{M}\right)+\mathrm{CH}_{3} \mathrm{OH} 0,15 \mathrm{M}+$ & \\
$\quad+0,2 \mathrm{M} \mathrm{NaOH}$ & 9.2 \\
$\mathrm{Pt} / \mathrm{Bi}^{3+}\left(1 \times 10^{-6} \mathrm{M}\right)+\mathrm{CH}_{3} \mathrm{OH} 0,15 \mathrm{M}+$ & 36.9 \\
$+0,2 \mathrm{M} \mathrm{NaOH}$ & \\
\hline
\end{tabular}
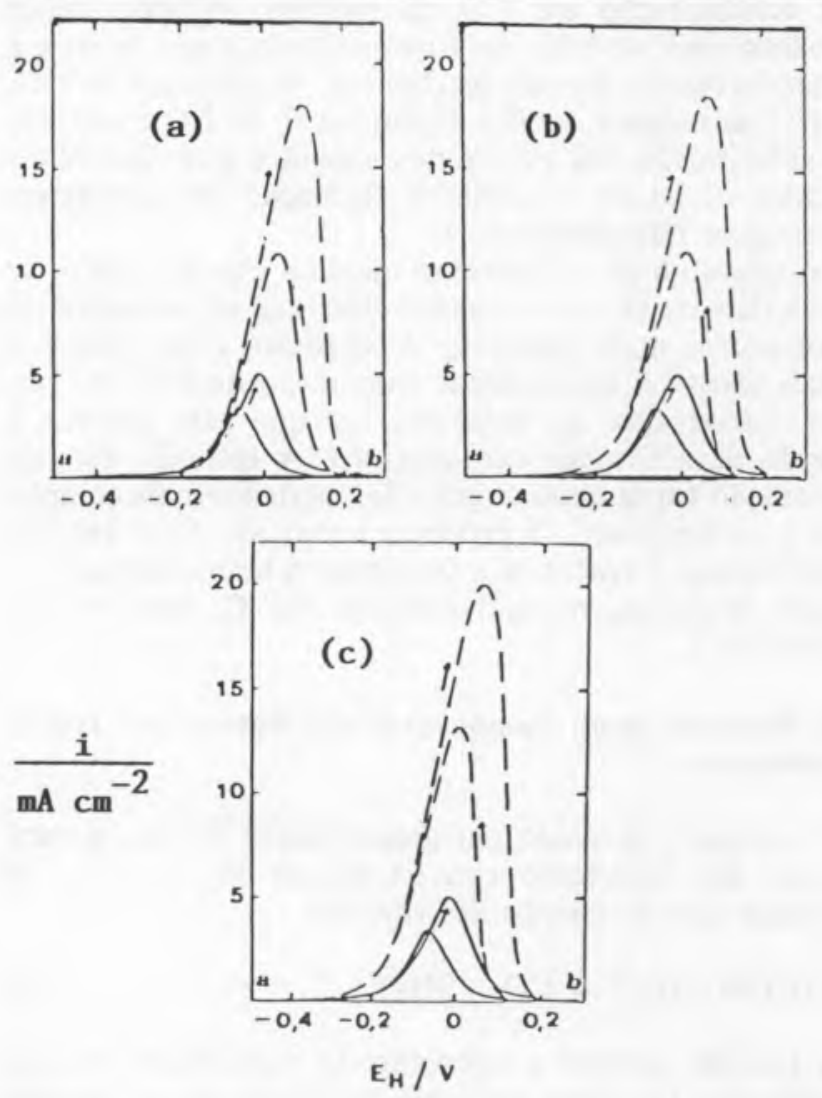

Fig. 2

Voltamogramas cíclicos do metanol em Pt na ausência (-) e na presença (--) de:
(a) $10^{-6} \mathrm{M} \mathrm{TlClO}_{4}$;
(b) $5 \times 10^{-6} \mathrm{M} \mathrm{Pb}\left(\mathrm{ClO}_{4}\right)_{2}$;
(c) $10^{-6} \mathrm{M} \mathrm{Bi}\left(\mathrm{ClO}_{4}\right)_{3}$.
$\mathrm{v}=50 \mathrm{mV} \mathrm{s}^{-1}$ (ref. 7).

As curvas da fig. 2 mostram bem o aumento da densidade de corrente, para um mesmo valor de potencial conseguido com a adição dos referidos iões metálicos sendo o efeito particularmente significativo para $\mathrm{E}=\mathrm{OV} v s$ E.N.H.

Os diferentes substratos têm actividade catalítica diferente, já que para um mesmo sobrepotencial $(\eta=0)$ produzem densidades de corrente $i_{o}$ bastante diferentes. O papel da electrocatálise, neste contexto é compreender as razōes e os factores que determinam essas diferenças de actividade catalítica, de modo a fabricar eléctrodos económicos e com uma capacidade elevada de produção de energia/por grama de catalisador e por dólar.

Também no nosso laboratório e no domínio da Electrocatálise estão em curso estudos sobre a oxidação electroquímica do Metanol em Pt modificada por adatomos.

Só a importância que o Metanol ganhou como combustível pode justificar o investimento que continua a 
ser feito pelos países mais ricos, na montagem e aperfeiçoamento de mais estaçð̌es produtoras de Metanol a partir do gás natural quando a produção, pelo menos de momento, é superior à procura no mercado.

\section{REFERÊNCIAS}

1. W.R. Grove, Phil. Mag., 14, 127 (1839).

2. B.D. Nicol, J. Electroanal. Chem., 118, 71 (1981).
3. W. Vielstich, Abstracts of the 36th ISE Meeting, Salamanca (1985).

4. M. Watanabe and S. Motoo, J. Electroanal. Chem., 60, 267 (1975); Ibid., 61, 429 (1976); Ibid, 209, 157 (1986).

5. Yu. B. Vassilev, V.S. Bagotzky, N.V. Osetrova and A.A. Mikahailova, J. Electroanal. Chem., 97, 63 (1979).

6. B. Beden, F. Kardigan, C. Lamy, and M. Leger, J. Electroanal. Chem., 127, 85 (1981).

7. G. Kokkinidis and D. Janna Koudakis, J. Electroanal. Chem., 153, 185 (1983).

8. G. Kokkinidis and G. Papanastasion, J. Electroanal. Chem., 221, 175 (1987).

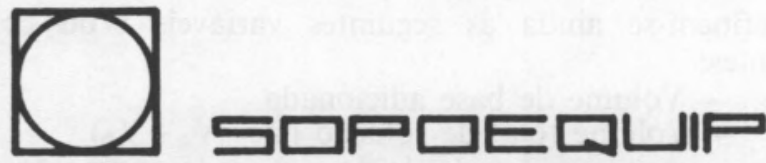

PRODUTOS E EQUIPAMENTOS PARA A INDÚSTRIA E LABORATORIOS LDA

\section{DINAMISMO - QUALIDADE \\ SERVIÇO}

\section{ESCOLHA - EFICIÊNCIA}

PEÇA-NOS A LISTA DAS NOSSAS REPRESENTADAS

ALGUMA LHE INTERESSARÁ!

Estamos à distância do seu telefone... 